\title{
Future Alcohol-Attributable Mortality in France Using a Novel Generalizable Age-Period-Cohort Projection Methodology
}

Sergi Trias-Llimós $\mathbb{1}^{1,2, *}$, Anastasios Bardoutsos ${ }^{1}$, and Fanny Janssen ${ }^{1,3}$

${ }^{1}$ Population Research Centre, Faculty of Spatial Sciences, University of Groningen, Landleven 1, 9747 Groningen, The Netherlands, ${ }^{2}$ Center for Demographic Studes, Centres de Recerca de Catalunya (CERCA), Carrer de Ca n'Altayó, Edifici E2, 08193 Bellaterra, Spain, and ${ }^{3}$ Netherlands Interdisciplinary Demographic Institute, KNAW/University of Groningen, Lange Houtstraat 19, 2511 The Hague, The Netherlands

*Corresponding author: Center for Demographic Studes, Centres de Recerca de Catalunya (CERCA), Carrer de Ca n'Altayó, Edifici E2, 08193 Bellaterra, Spain. Tel.: 0034 935813060; E-mail: strias@ced.uab.cat

Received 11 May 2020; Revised 22 September 2020; Editorial Decision 22 September 2020; Accepted 22 September 2020

\begin{abstract}
Aim: To forecast age- and sex-specific alcohol-attributable mortality in France for the period 20152050 using a novel generalizable methodology that includes different scenarios regarding period and cohort change.

Methods: For the French national population aged 25-90 years (1979-2014), we estimated alcoholattributable mortality by mortality from the main causes of death wholly attributable to alcohol, plus liver cirrhosis mortality. We modelled sex-specific alcohol-attributable mortality by adjusting for age, period and birth cohort. We forecasted the model parameters to obtain future age- and sex-specific alcohol-attributable mortality up until 2050 using a conventional baseline, scenario I (favourable period change) and scenario II (unfavourable cohort change).

Results: Alcohol-attributable mortality is clearly declining in France, with the decline decelerating from 1992 onwards. In 2014, the age-standardized alcohol-attributable mortality rates, in deaths per 100,000 , were 34.7 among men and 9.9 among women. In 2050, the estimated rates are between 10.5 (prediction interval: 7.6-14.4; scenario I) and 17.6 (13.1-23.7; scenario II) among men, and between 1.1 (0.7-1.7; scenario I) and 1.8 (1.2-2.9; scenario II) among women; which implies declines of $58 \%$ for men and $84 \%$ for women (baseline).

Conclusion: Alcohol-attributable mortality in France is expected to further decline in the coming decades, accompanied by age pattern changes. However, France's levels are not expected to reach the current lower levels in Italy and Spain for 15 years or more. Our results point to the value of implementing preventive policy measures that discourage alcohol consumption among people of all ages, but especially among adolescents.
\end{abstract}

\section{INTRODUCTION}

Alcohol consumption is a major public health issue in Europe (Franco 2015; WHO 2019), where the levels of both alcohol consumption and attributable mortality are the highest worldwide (WHO 2019). 
attributable mortality, and, consequently, the impact of alcohol on overall mortality. However, there is a lack of knowledge about future trends in alcohol-attributable mortality.

When estimating future alcohol-attributable mortality, it is essential to understand past trends, which differ widely across European countries depending on their drinking cultures. Alcoholattributable mortality has been gradually decreasing in Southern Europe since around the 1970s, but the long-term trends in other European countries have been more irregular (Kraus et al., 2015; Trias-Llimós et al., 2017). In examining past trends in alcoholattributable mortality, birth cohorts must be taken into account (Kraus et al., 2015; Trias-Llimós et al., 2017). Birth cohort differences in the adoption of unhealthy lifestyles, including heavy drinking, are often attributed to certain birth cohorts facing similar contextual situations at different ages than other birth cohorts. In addition, we know that alcohol use in younger adulthood is associated with overall drinking patterns over the life course (Pitkänen et al., 2005) and with the development of alcohol-related problems (Hingson et al., 2006; Dawson et al., 2008). Thus, when forecasting alcohol-attributable mortality, it may be useful to take birth cohorts into account.

Despite the important role birth cohorts play in determining alcohol-attributable mortality trends, only one previous study barely touched upon this dimension when discussing future levels of alcoholattributable mortality (Rosén and Haglund 2019). By assuming age effects and cohort effects to be constant over time, which is arguable over the short term, it has been projected that alcoholattributable mortality will decline moderately in Sweden between 2015 and 2025 (Rosén and Haglund 2019). Other efforts to project alcohol-attributable mortality overlooked the cohort dimension. For example, Pruckner et al. (2019) linearly projected declines between 1979 and 2015 in age-standardized death rates for selected alcoholrelated causes of death up until 2030 for 29 European countries, but they did not account for the cohort dimension. Other projections of alcohol-attributable mortality failed to simultaneously account for both period and cohort effects. Sheron et al. $(2011,2012)$ used linear extrapolation techniques to project alcohol-attributable liver deaths in the UK up to 2030 while also applying past time trends from other European countries to the UK. Thus, projections of future alcoholattributable mortality are currently available for two countries only and for relatively short time periods. It should also be noted that in addition to being based on arguable assumptions; these results were not provided disaggregated by age group.

We aim to forecast overall and age-specific alcohol-attributable mortality in France by sex over a long period (2014-2050) using age-period-cohort (APC) modelling while including different likely scenarios for the period and cohort dimensions. To do so, we have used a methodology that can be applied to other European countries.

France is a country of particular interest as, even though alcohol consumption has declined in France in recent decades (Gual and Colom 1997; WHO 2019), its current levels of alcohol consumption are still high (WHO 2019). Moreover, France has higher levels of alcohol-attributable mortality than most other Western European countries (Trias-Llimós, Kunst, et al., 2018). According to recent estimates for France, $13 \%$ of deaths among men and $5 \%$ of deaths among women are attributable to alcohol (Guerin et al., 2013). Finally, in order to carefully study past trends and to adequately capture cohort effects, information about long-term trends in alcohol-attributable mortality is needed. However, because of changes in the International Classification of Diseases (ICD) (Janssen and Kunst 2004), these trends cannot be directly estimated from cause-of-death data from the international WHO Mortality
Database. More detailed national cause-of-death data, available for France, are therefore required.

\section{MATERIALS AND METHODS}

\section{Setting and study population}

For the total population of men and women in France aged 25-90, we studied past trends in overall and age-specific alcohol-attributable mortality (1979-2014) and estimated future overall and age-specific alcohol-attributable mortality (2015-2050).

\section{Data}

We used detailed cause-of-death data by single year of age and sex for the 1979-2014 period from the Center for Epidemiology on the Medical Causes of Death (CépiDc) at the French National Institute of Health and Medical Research (Inserm) (Rey 2016). In line with previous studies, alcohol-attributable mortality was defined as the sum of deaths from the main causes wholly attributable to alcohol (mental and behavioural disorders due to alcohol use, alcoholic liver disease and alcohol poisoning) and liver cirrhosis deaths (ICD-9 codes: 291, 303, 571 and E860-E866; ICD-10 codes: F10, G31.2, K70, K73, K74 and X45), as similarly done in previous studies (Jasilionis et al., 2011; Trias-Llimós, Martikainen, et al., 2018). We used the corresponding population data from the Human Mortality Database to estimate alcohol-attributable mortality rates (HMD 2017).

\section{Approach}

We applied a generalized methodology to forecast (age-specific) alcohol-attributable mortality that uses APC modelling and includes different likely scenarios for the period and cohort dimensions. A crucial part of this methodology is the careful assessment of past trends prior to the formulation of the assumptions for the baseline projection and the likely scenarios based on observed data. In the remainder of the methodology section, we will describe the methodology used to study past trends (APC modelling), and describe the methodology and assumptions used to estimate future alcoholattributable mortality levels and trends (projection methodology).

\section{APC modelling}

We estimated yearly sex-specific age-standardized alcohol-attributable mortality, using the total French population of 2014 as the standard. We modelled alcohol-attributable mortality by means of APC modelling

$$
\log \mu_{x, t}^{\mathrm{alc}}=\alpha_{x}+\kappa_{t}+\gamma_{t-x},
$$

where $\mu_{x, t}^{\text {alc }}$ are alcohol attributable mortality rates by age $(x)$ and year $(t)$. The parameters $\alpha_{x}, \kappa_{t}$ and $\gamma_{t-x}$ capture the age pattern, the overall time trend (period) and the cohort patterns, respectively. We assume that alcohol-attributable deaths follow a Poison distribution.

To deal with the linear dependency between period and birth cohort (age $=$ period - cohort $)$ in the APC modelling, we used the approach by Cairns et al. (2009). This method builds on the commonly applied Clayton and Schifflers APC approach (Clayton and Schifflers 1987), which distinguishes between period, cohort and drift. One additional step is performed to move the drift (=shared linear trend between period and cohort) completely to either the period or the cohort trend. This is especially beneficial when the aim is to extrapolate past trends because you end up with two time-dependent factors that are easy interpretable and that can meaningfully be 
extrapolated into the future (Janssen et al., 2020). In our application of the Cairns et al.'s approach, we moved the drift to the period trend. The period parameter thus captures the entire linear time trend (including the drift), while the cohort parameter captures the cohort variations from this overall trend (see (Cairns et al., 2009) for further details). We used, for this purpose, the Stochastic Mortality Modelling (StMoMo) package (Villegas et al., 2015) in R (R Core Team 2018).

To ensure the robustness of the cohort estimates, we excluded from the model the first and the last five birth cohorts, which were highly uncertain (wide confidence intervals) due to the reduced number of available data points (see Fig. S1). This resulted in the inclusion of the cohorts born between 1894 and 1984.

\section{Projection methodology}

Based on the past trends analysis (see results section-past trends), we formulated a baseline projection and two likely scenarios. The baseline projection used common settings in APC mortality projections and in general (mortality) forecasting. That is, we assumed that the birth cohort parameter was constant at the mean observed over the past period (Stoeldraijer et al., 2015; Rosén and Haglund 2019). For the period parameter, we considered the trend for the most recent period (1992-2014), which represents the period after the clear trend break in 1992 (see Fig. 3) (Janssen and Kunst 2007; Sheron et al., 2011).

Scenarios I (period) and II (cohort) account for likely changes in the period and the cohort dimension, respectively, relative to the baseline projection. Scenario I represents a positive period change (stronger decline), whereas scenario II is a negative cohort scenario. In scenario I (period), we assumed a more pronounced decline in alcohol-attributable mortality than in the more recent period, given the possibility that favourable contextual changes or more effective preventive policies will favourably affect the drinking behaviour of people in all age groups. This is an optimistic, yet plausible, scenario given the growing awareness of the health damage of alcohol consumption (Rehm et al., 2017), and the potential of contextual and policy changes to reduce alcohol use among adolescents (Noel 2019) and in France (Cogordan et al., 2014). In addition, France has currently higher alcohol consumption levels as compared with similar countries (Spain and Italy) (WHO Global Health Observatory Data Repository, n.d.), and therefore higher possibilities for reducing alcohol consumption. In this scenario, we therefore extrapolate the stronger declines over the whole 1979-2014 period compared with the less strong declines over the more recent period (1992-2014).

In scenario II (cohort), we assumed that the increase in mortality among the most recent cohorts (as seen in Fig. 1) will also have negative effects on mortality in the subsequent birth cohorts, given the possibility that negative changes in drinking behaviour (and especially binge drinking) will continue across generations of young people. This scenario is supported by the evidence suggesting that drinking patterns are becoming riskier among young generations (Richard et al., 2015), which can also explain the increase in mortality among the most recent cohorts. Nonetheless, recent evidence suggests that alcohol consumption among young adults has been declining over time (Com-Ruelle and Le Guen 2013; WHO 2018). Therefore, we assumed elevated-but converging-mortality levels compared with earlier cohorts for-at least-the future 35 cohorts. We implemented this in our model by allowing the cohort parameter to gradually converge towards the mean, instead of being fixed at the mean.
For each scenario, we forecasted the period and cohort parameter by using autoregressive integrated moving average (ARIMA) time series models. We selected the best ARIMA model based on the corrected Akaike's information criterion (Hurvich and Tsai 1989) and the Hyndman-Khandakar algorithm (Hyndman and Khandakar 2007). For the implementation of the algorithm, we used the auto.arima function from package forecast in R (R Core Team 2018). See Table 1 for further details on the specific time series choices for the different scenarios.

We projected age-specific alcohol-attributable mortality rates up to 2050 for the baseline and the two scenarios and estimated the respective $95 \%$ prediction intervals by performing 10,000 simulations. The projected rates were subsequently age-standardized using the total French population of 2014 as the standard.

\section{RESULTS}

\section{Past trends}

Alcohol-attributable mortality was clearly declining in France (19792014), but with two distinct trends: (a) a pronounced decline from 1979 to 1992 and (b) a slower decline from 1992 to 2014, especially among men (Fig. S2).

When looking at the period trend in our APC model, we see that the decline was larger among women than among men (see Fig. 1). When examining the general decline in alcohol-attributable mortality over time, we observe that alcohol-attributable mortality increased among the cohorts born before 1955, then decreased among the cohorts born between 1955 and 1975, and increased for the more recent birth cohorts (Fig. 1).

\section{Future trends}

The results for the projected period and birth cohort parameter for the different scenarios are shown in Fig. 2.

The results showed that the age-standardized alcohol-attributable mortality rates will continue to decline for both men and women, albeit at different speeds depending on the period and cohort assumptions (Fig. 3, Table S1 for the prediction intervals).

The baseline scenario projected a decline in age-standardized alcohol-attributable mortality, by deaths per 100,000, from 34.7 in 2014 to 14.7 (prediction interval: 11.2-19.6) in 2050 among men and from 9.9 in 2014 to $1.6(1.0-2.4)$ in 2050 among women; or by $58 \%$ among men and $84 \%$ among women. The gender gap in alcoholattributable mortality is projected to narrow over time in absolute terms, from 25 deaths per 100,000 in 2014 to 13 in 2050 (baseline scenario).

Scenario I (favourable period change) obviously resulted in larger declines and lower future age-standardized alcohol-attributable mortality in 2050: 10.5 (7.6-14.1) among men and 1.1 (0.7-1.7) among women. Scenario II (unfavourable cohort change) resulted in smaller declines and higher future levels in 2050: 17.6 (13.1-23.7) among men and 1.8 (1.2-2.9) among women. As scenario II estimated higher mortality for the currently young generations only, but cohort mortality that is almost identical to the baseline projection (same period assumptions) in the long run (e.g. cohorts born in 2020), the overall estimates from the baseline projection and from scenario II tended to converge in the long run (Fig. S3).

Whereas in 2014, an inverse u-shaped age pattern of alcoholattributable mortality was observed with a peak at around age 65, this peak is projected in each projection scenario to occur at higher ages among men up to about 2040 , and among women at least up 

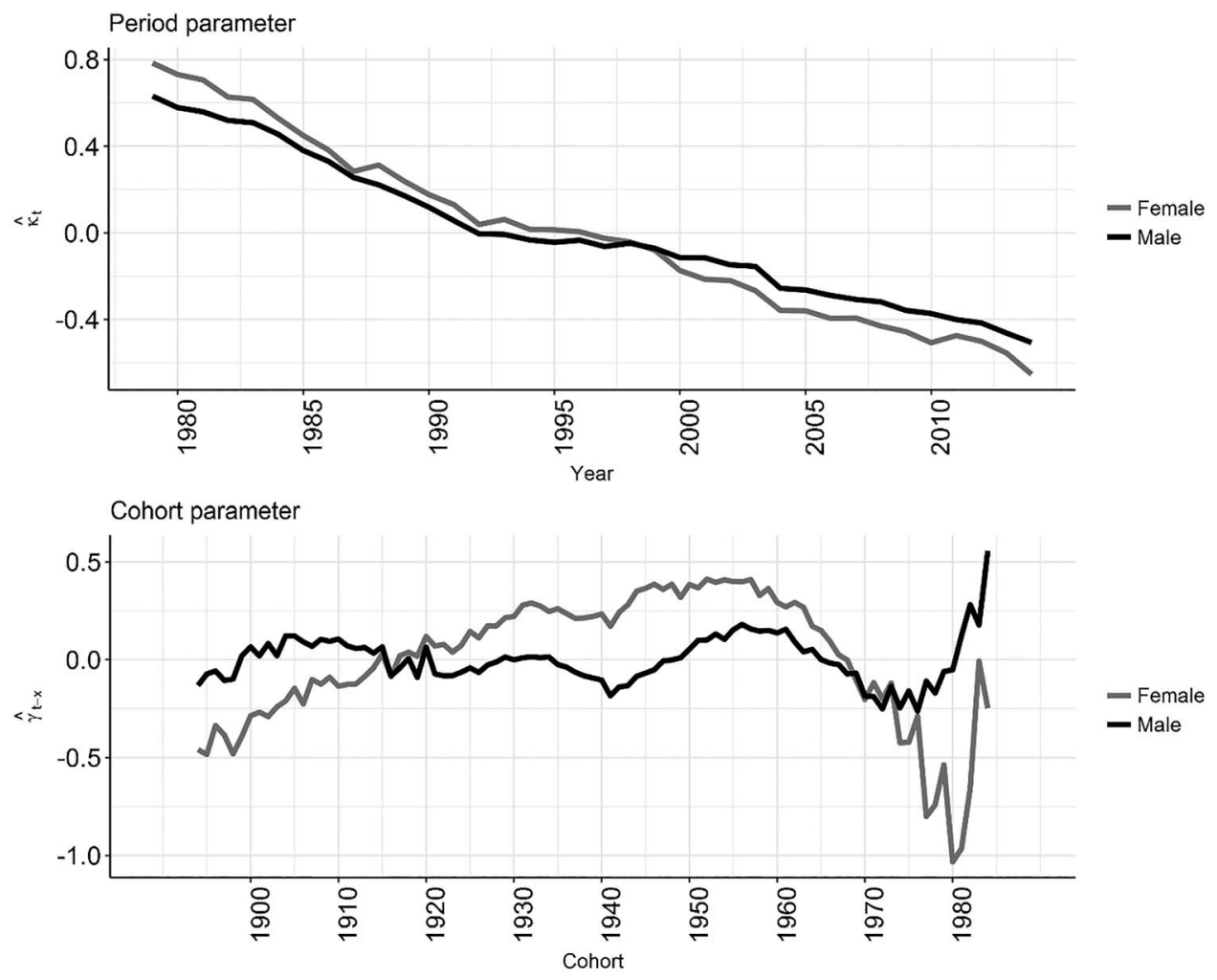

Fig. 1. Period and (non-linear) birth cohort effects of alcohol-attributable mortality in France (ages 25-90, 1979-2014), by sex.

Table 1. Summary of scenarios and time-series specifications for the projection of the period and cohort parameters

\begin{tabular}{|c|c|c|c|c|}
\hline Projection & $\begin{array}{l}\text { Period parameter } \\
\left(\kappa_{t}\right)\end{array}$ & $\begin{array}{l}\text { Time series } \\
\text { specification period }\end{array}$ & Cohort parameter $\left(\gamma_{t-x}\right)$ & $\begin{array}{l}\text { Time series } \\
\text { specification cohort }\end{array}$ \\
\hline Baseline & $1992-2014$ & ARIMA $(0,1,0)$ & $\begin{array}{l}\text { Men: constant along the mean }(0) \text {. } \\
\text { Women: constant along the mean } \\
\text { for the last } 10 \text { cohorts }(-0.56) \text {. }\end{array}$ & ARIMA $(0,0,0)$ \\
\hline Scenario I (period) & $1979-2014$ & ARIMA $(0,1,0)$ & $\begin{array}{l}\text { Men: constant along the mean }(0) \text {. } \\
\text { Women: constant along the mean } \\
\text { for the last } 10 \text { cohorts }(-0.56) \text {. }\end{array}$ & ARIMA $(0,0,0)$ \\
\hline Scenario II (cohort) & $1992-2014$ & ARIMA $(0,1,0)$ & $\begin{array}{l}\text { Men: gradually converging } \\
\text { towards the mean. } \\
\text { Women: same pattern as for men, } \\
\text { but shifted downwards based on } \\
\text { the differences in the mean } \\
\text { between men and women (last } 10 \\
\text { birth cohorts). }\end{array}$ & ARIMA $(1,0,2)$ \\
\hline
\end{tabular}

*For women, unlike as for men, the average for the most recent birth cohorts is substantially lower than the overall average (zero). In that case, we assumed the mean to be the mean over the last 10 birth cohorts.

until 2050, which results in a linear increase with age for women in 2050 (Fig. 4). Furthermore, a second sharp peak in the age pattern, which occurs at higher ages in later years, was observed. This peak was especially visible in the baseline projection and period scenario, in which the cohort parameter was set to the mean observed over the past period, in line with common settings in APC mortality projections (Stoeldraijer et al., 2015; Rosén and Haglund 2019). In the cohort scenario, the peak was more extended and more realistic, as here it was assumed that cohort mortality would gradually decline towards the mean.

\section{DISCUSSION}

\section{Summary of results}

Alcohol-attributable mortality was clearly declining among the French population aged 25-90 in the 1979-2014 period, with a deceleration of the decline from 1992 onwards. In 2014, the agestandardized rates, in deaths per 100,000, were 34.7 among men and 9.9 among women. Over the 2014-2050 period, the age-standardized alcohol-attributable mortality rates are projected to further decline by $58 \%$ for men and $84 \%$ for women (baseline). This implies a 

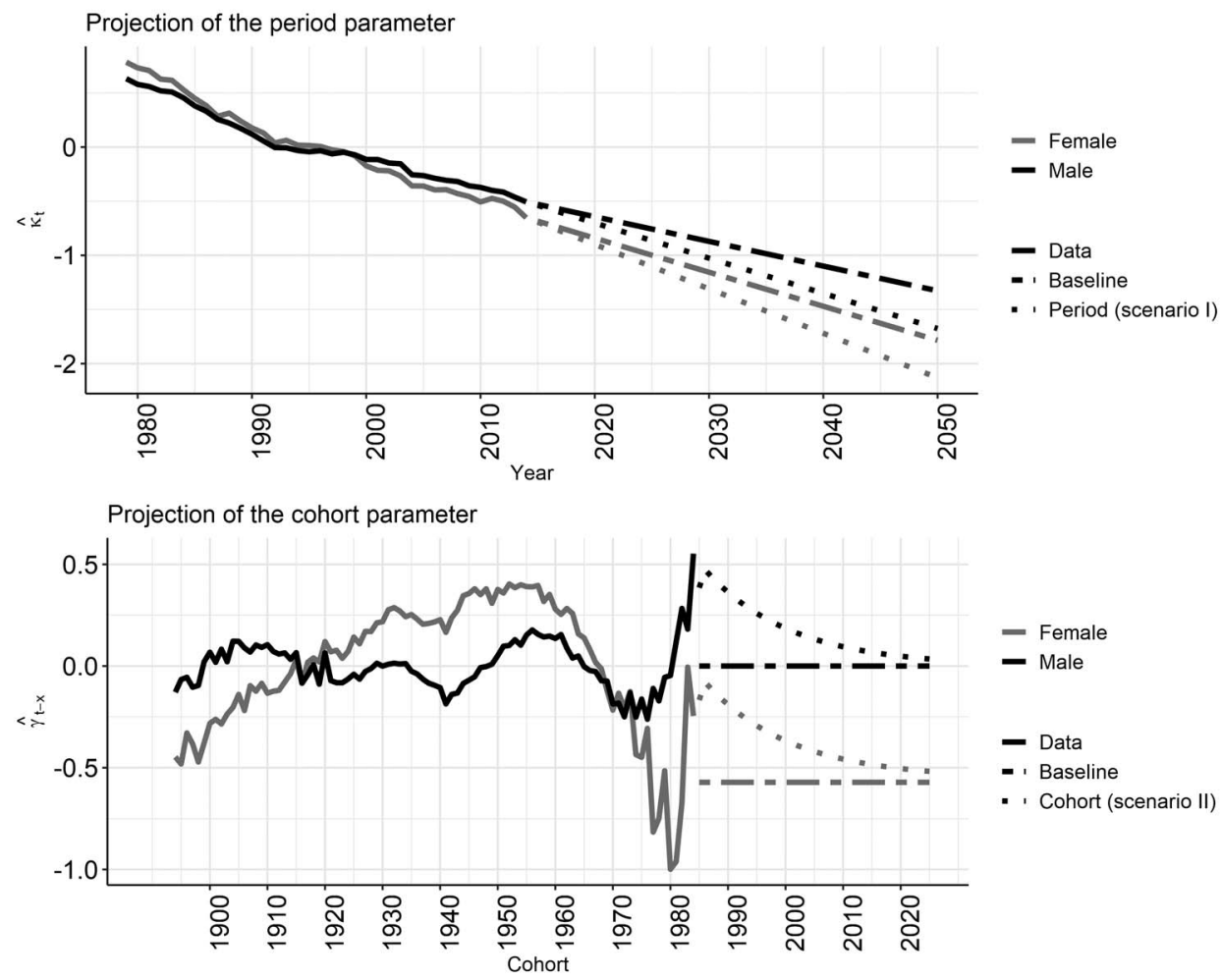

Fig. 2. Alcohol-attributable mortality in France (ages 25-90): age, period and cohort parameters and projections of period and cohort parameters, by sex.

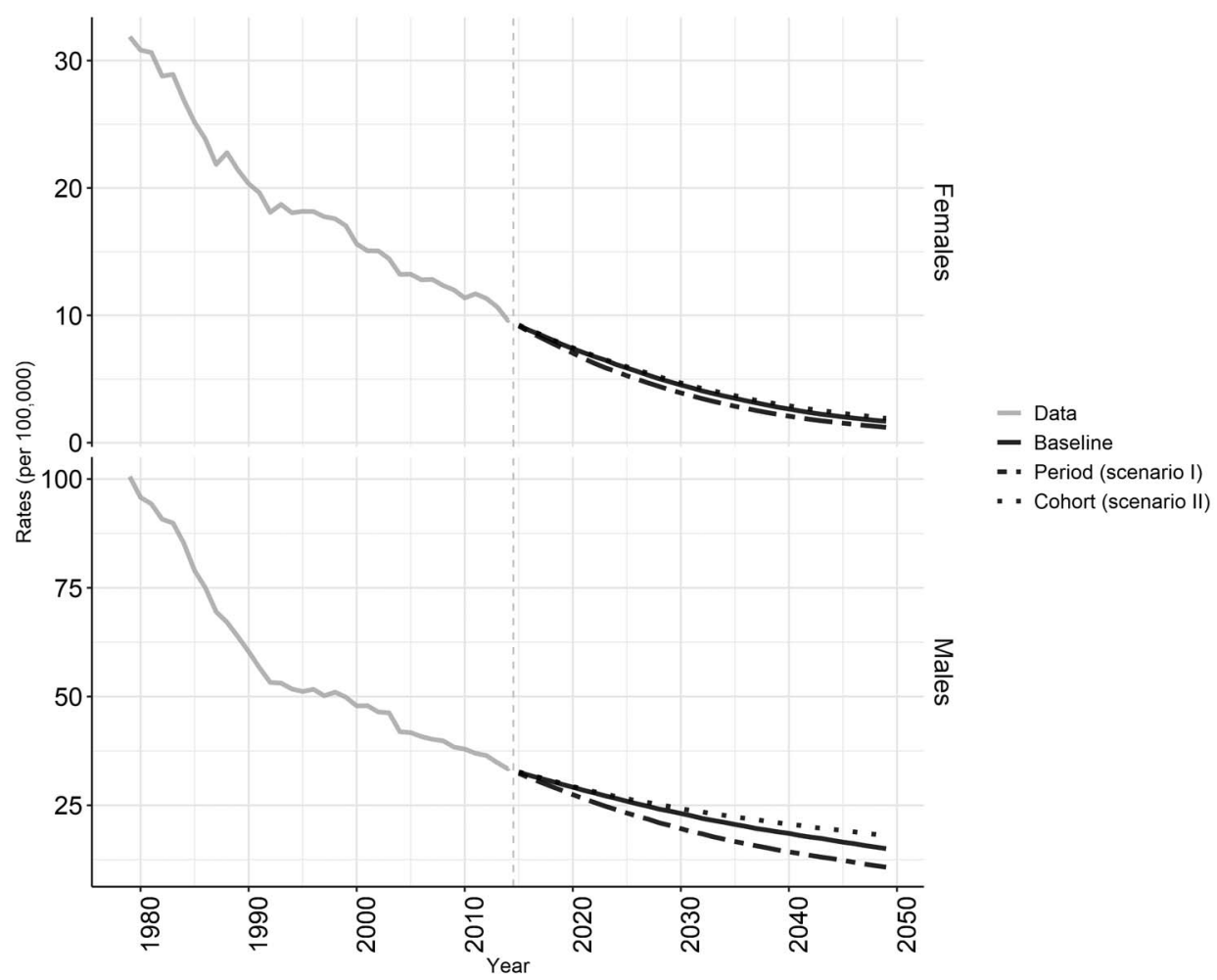

Fig. 3. Age-standardized alcohol-attributable mortality rates (ages 25-90) in France (1979-2014 data; 2015-2050 projection), by sex. 


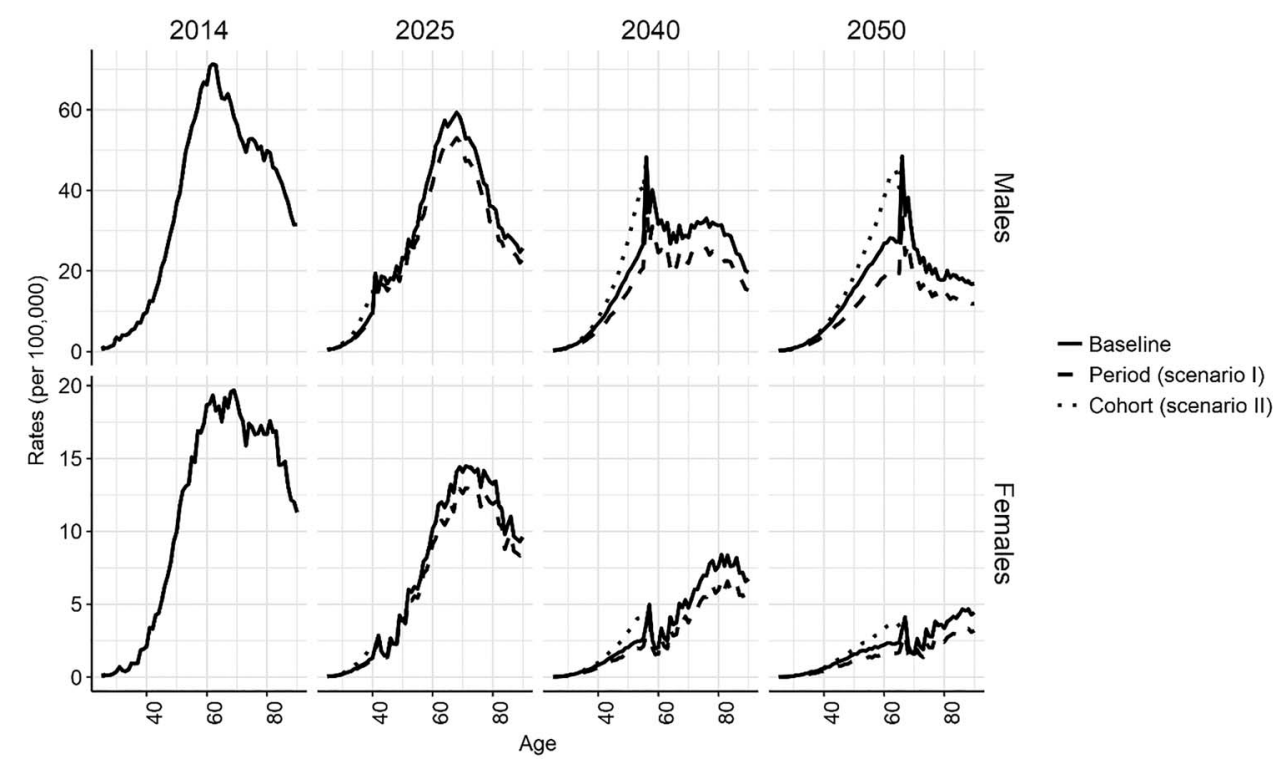

Fig. 4. Observed $(2014)$ and projected $(2025,2040,2050)$ age-specific alcohol-attributable mortality rates (25-90) in France, by sex.

range in 2050 , in deaths per 100,000 , of between 10.5 (7.6-14.4; scenario I period) and 17.6 (13.1-23.7; scenario II cohort) among men, and between 1.1 (0.7-1.7; scenario I period) and 1.8 (1.2-2.9; scenario II cohort) among women. The peak of the inverse u-shaped age pattern of alcohol-attributable mortality (currently at around age 65 ) is expected to shift towards older ages, and an additional hump in the age pattern is projected that moves towards higher ages with time, and is more extended in the cohort scenario.

\section{Reflection on data}

In line with previous research, we used the main causes of death wholly attributable to alcohol, supplemented with liver cirrhosis mortality, to estimate alcohol-attributable mortality (Jasilionis et al., 2011; Trias-Llimós, Martikainen, et al., 2018). The selected causes of death wholly attributable to alcohol and liver cirrhosis (F10, G31.2, K70, K73, K74, and X45) represent $>90 \%$ of the total deaths wholly attributable to alcohol in France (Trias-Llimós, Martikainen, et al., 2018). Data for the remaining very specific causes of death wholly attributable to alcohol (Rehm et al., 2017) were not available to us. Also, the inclusion of liver cirrhosis mortality as a whole, which is mostly-but not always-driven by alcohol (Trias-Llimós et al., 2017), was necessary as it was not possible to build coherent time trends purely for alcoholic liver cirrhosis. Overall, our estimate of alcohol-attributable mortality underestimates total alcohol-attributable mortality (Trias-Llimós, Martikainen, et al., 2018) because alcohol has a notable impact on other causes as well, such as ischemic diseases, cancers and injuries (Rey et al., 2010; Guerin et al., 2013; Rehm et al., 2017). The extent of this underestimation in 2010 for France was estimated to be around $58 \%$ relative to estimates using attributable fraction methods, and between $27 \%$ (women) and $42 \%$ (men) relative to estimates from approaches using underlying and contributory causes of death (Trias-Llimós, Martikainen, et al., 2018). Therefore, the results of this paper on alcohol-attributable mortality levels should be interpreted as strictly referring to past and future mortality due to causes wholly attributable to alcohol and liver cirrhosis.

The past and future time trends in alcohol-attributable mortality are, however, much less affected by our estimation. First, the past mortality trends from the selected causes of death, and especially liver cirrhosis, followed past overall alcohol consumption trends (Ramstedt 2001). Second, our method for estimating alcohol-attributable mortality seems to have a rather minor effect on time trends if we compare our data to the Global Burden of Diseases (GBD) estimates, which are based on population attributable fraction approaches (Forouzanfar et al., 2016). That is, the overall decline in alcoholattributable mortality between 1990 and 2013 among men is 40.5\% in the GBD estimates (Trias-Llimós, Kunst, et al., 2018), and 41.7\% in our study ( 36.5 and $47.3 \%$ for women, respectively). Therefore, our estimates of the past and future alcohol-attributable mortality decline can be considered good approximations of the overall decline in alcohol-attributable mortality in relative terms. Explanation of results. The projected future decline in alcohol-attributable mortality is based on the extrapolation of past declines in alcohol-attributable mortality. These past declines in alcohol-attributable mortality can be mainly attributed to declines in overall alcohol consumption in France, from 19.41 of pure alcohol consumption per capita and year in 1979 to 12.01 in 2014 (WHO Global Health Observatory Data Repository, n.d.). These declines were driven by an overall reduction in daily wine consumption (Gual and Colom 1997; WHO 2019) in a context of societal and economic changes (e.g. urbanization and increased female employment) (Cogordan et al., 2014). In that sense, we assume that societal and economic change will lead to a continued decline in alcohol-attributable mortality in the future.

Our APC analysis however also revealed a potential important deviation from the overall decline. That is, the most recent birth cohorts showed relatively higher alcohol-attributable mortality than their older counterparts. This relates as well to trends in alcohol consumption. Alcohol consumption among adolescents and young adults have also been moderately declining over time (Com-Ruelle and Le Guen 2013; WHO 2018), and therefore contributed as well to the observed decline in alcohol-attributable mortality over time. However, recent trends for self-reported drunkenness episodes suggested increases among 18-25 year old from 2005 to 2015 (Richard et al., 2015) and a stagnation or a moderate decline among 15 -yearolds (WHO 2018). These more harmful drinking patterns are likely 
the result of a complex combination of determinants, including an earlier onset of drinking and unfavourable changes in social norms (WHO 2018). At young ages, alcohol-related mortality is driven mostly by external causes of death (Trias-Llimós, Martikainen, et al., 2018), which are more closely related with drinking patterns (e.g. drunkenness) (compared with overall alcohol consumption levels) (Rehm et al., 2017). Explanations for this complex trend include age at drinking onset, family life and societal norms and rules (WHO 2018). These somewhat contrasting trends among young individuals (a decrease in overall consumption, and an increase of riskier drinking patterns) suggest that adolescents drink less often, but more harmfully. We incorporated the potential increase in riskier drinking patterns in our cohort scenario, leading to-still—a general decline in alcohol-attributable mortality but to elevated risks among younger birth cohorts and-as a result-a changing age pattern of alcohol-attributable mortality.

Despite the overall expected further decline in alcohol-attributable mortality in France, it might take some time before France reaches the currently observed lower levels of alcohol-attributable mortality in Spain and Italy. In fact, we project that France will not reach Spain's current levels of alcohol-attributable mortality (22.0 deaths per 100,000) until 2032 and will not reach Italy's current levels (17.5 deaths per 100,000) before 2040 (Fig. S4). This is because France historically has had and currently still has higher levels of both alcohol consumption per capita and attributable mortality (Trias-Llimós, Kunst, et al., 2018). This can be related, in particular, to the higher wine and spirits consumption in France as compared with Spain and Italy (WHO Global Health Observatory Data Repository, n.d.). According to our baseline projection for men, and using the same definition for alcohol-attributable mortality, France will not reach Spain's current levels (22.0 deaths per 100,000) until 2032 and will not reach Italy's current levels (17.5 deaths per 100,000) before 2040 (see Fig. S4). Thus, even though alcohol-attributable mortality is expected to further decline in France, it will not decrease to the current levels in these neighbouring countries in the next decade.

In addition to a further decline in alcohol-attributable mortality, we predict a changing age pattern. In particular, we project that the peak of the inverse $\mathrm{u}$-shaped age pattern of alcohol-attributable mortality (currently at around age 65) will shift towards older ages. For men, this seems to be a temporal phenomenon only (up to about 2040), whereas for women, it lasts at least up until 2050, with the peak expected to occur at age 85 . For example, the cohorts born around 1955 (aged 70 in 2025 and aged 85 in 2040), and especially the women in these cohorts, are at higher risk of alcohol-attributable mortality than other observed cohorts (Fig. 2), as the observed age patterns show (Fig. 4). In both cases, this is entirely explained by the cohort effects. Thus, these relative changes in age patterns are not observed in the very long term, when cohort differences tend to be zero (see Fig. S5).

Furthermore, the additional humps in the projected age pattern can be entirely explained by cohort effects. That is, for each subsequent period, this hump occurs at a higher age (at age 42 for 2025, at age 57 for 2040, at age 67 for 2050), at least for the baseline projection and the period scenario. The birth cohorts causing this pattern are the youngest birth cohorts we observed; i.e. those born in the early 1980s. The finding that alcohol-attributable mortality is higher among the younger generations than among the preceding generations could be explained by the increase of riskier drinking patterns (e.g. drunkenness) among the generations born in the 1980s and early 1990s (Richard et al., 2015). For the baseline projection and the period scenario, this represents a very abrupt peak, mainly because mortality for the younger observed cohorts is higher than the average level, and because we assumed that the younger cohorts will have the same cohort mortality as the average level of the previous cohorts, as commonly done (Rosén and Haglund 2019). In our cohort scenario, we instead adopted a more gradual change of the cohort parameter, which is likely to result in an extended hump and reflects the decline in alcohol use observed among the generations born in late 1990s and 2000s (WHO 2018).

\section{Appraisal of the methodology}

To forecast (age-specific) alcohol-attributable mortality, we applied a methodology that uses APC modelling and includes different likely scenarios for the period and cohort dimensions.

Our main rationale for applying an APC projection methodology was that previous research demonstrated that the birth cohort dimension has clear added value in describing and understanding past alcohol-attributable mortality trends (Kraus et al., 2015; Trias-Llimós et al., 2017). Moreover, in previous forecasts of alcoholattributable mortality (Rosén and Haglund 2019) and of smokingattributable mortality (Stoeldraijer et al., 2015), APC projection was applied using the same rationale. Our 'explanation of results' section shows the added value of including the cohort dimension when forecasting alcohol-attributable mortality, as cohort effects were found to have a substantial impact on the projected age pattern in alcohol-attributable mortality at the population level.

To deal with the linear identification problem $($ period $=$ cohort + age) in APC modelling, we applied the approach developed by Cairns et al. (2009). Their approach allows to allocate the complete linear trend (including the drift) to the period parameter in a straightforward manner, leaving the cohort parameter as purely non-linear, which is beneficial when projecting (Janssen et al., 2020). Other approaches presented in the literature deal with the linear identification problem in other, mostly more complicated, ways (Yang et al., 2004; Carstensen 2007). Generally, different APC approaches generate similar outcomes when applied to past trends (Yang et al., 2004). One could therefore also use a different APC model as part of our general projection methodology. Applying models with multiple period or cohort parameters, however, will require more assumptions, could make projections less transparent and could result in different projection outcomes.

In contrast to the only previous projection of alcohol-attributable mortality that included the cohort dimension (Rosén and Haglund 2019), we considered different assumptions for both period and birth cohort based on a careful study of past trends. Whereas the assumption of the period had an effect on the overall levels (in this study, lower mortality levels), the assumption of the cohort had an effect on both the overall levels (in this study, higher mortality levels) and the age pattern of alcohol-attributable mortality. The application of our approach allowed us to provide new insights into the importance of the assumptions for both overall and agespecific future alcohol-attributable mortality estimates. In particular, our results showed that the use of a constant cohort pattern, which is often recommended in the literature, may result in an abruptand unlikely-hump in the projected age pattern. Thus, for future APC projections, we recommend that more realistic assumptions are used for the continuation of the cohort parameter. To make such assumptions, a careful study of past trends is essential.

Our APC approach, which used different assumptions for the period and cohort dimensions, can be generalized and applied to 
other populations. However, to make logical assumptions for the projection and achieve realistic results for the future, examining past trends for the population under study is crucial.

\section{CONCLUSION}

In conclusion, alcohol-attributable mortality in France is expected to further decline in the coming decades, although France is not expected to reach the current lower levels of neighbouring countries with similar drinking cultures within the next 10 years. Furthermore, the peak of the inverse u-shaped age pattern of alcohol-attributable mortality (currently at age 65) is expected to shift towards older ages because alcohol-attributable mortality is higher among cohorts born around 1955 than among the other observed cohorts (except the male cohorts born in the early 1980s).

Given its profound effects on future mortality trends and the unfavourable age patterns for current generations, health policymakers should seek to prevent elevated alcohol-attributable mortality in the medium term by tackling alcohol consumption among adolescents (cohort measures). In addition, the implementation of preventive policy measures that affect people in all age groups (period measures; i.e. taxation) (Anderson et al., 2009) could positively contribute to alcohol-attributable mortality declines in the short term.

\section{SUPPLEMENTARY MATERIAL}

Supplementary material is available at Alcohol and Alcoholism online.

\section{AVAILABILITY OF DATA AND MATERIAL}

The data were obtained from the following sources: Inserm CépiDc (contact persons: Mireille EB, Grégoire Rey) and Human Mortality Database (publicly available at www.mortality.org).

\section{AUTHORS' CONTRIBUTIONS}

All authors designed the study. S.T.L. and A.B. carried out the empirical analyses. S.T.L. drafted the manuscript. F.J. aided in drafting the manuscript and critically reviewed the manuscript. All authors aided interpreting the results, and approved the final manuscript.

\section{ACKNOWLEDGEMENTS}

The authors thank Mireille EB and Grégoire Rey (Inserm-CépiDc, France) for sharing data with us. The authors are grateful to Leo van Wissen (University of Groningen and Netherlands Interdisciplinary Demographic Institute) and to Joop de Beer (Netherlands Interdisciplinary Demographic Institute) for their useful comments on an earlier version of this work.

\section{FUNDING}

This work is financed by the Netherlands Organisation for Scientific Research (NWO) in relation to the research programme 'Smoking, alcohol, and obesity, ingredients for improved and robust mortality projections', grant no. 452-13001. See www.futuremortality.com.

\section{CONFLICT OF INTEREST STATEMENT}

The authors declare that they have no competing interests.

\section{ETHICS APPROVAL AND CONSENT TO PARTICIPATE}

Not applicable.

\section{CONSENT FOR PUBLICATION}

Not applicable.

\section{REFERENCES}

Anderson P, Chisholm D, Fuhr DC. (2009) Effectiveness and cost-effectiveness of policies and programmes to reduce the harm caused by alcohol. Lancet 373:2234-46

Cairns AJ, Blake D, Dowd K, et al. (2009) A quantitative comparison of stochastic mortality models using data from England and Wales and the United States. North Am Actuar J 13:1-35.

Carstensen B. (2007) Age-period-cohort models for the lexis diagram. Stat Med 26:3018-45.

Clayton D, Schifflers E. (1987) Models for temporal variation in cancer rates. II: age-period-cohort models. Stat Med 6:469-81.

Cogordan C, Kreft-Jaïs C, Guillemont J. (2014) Effects of alcoholic beverage control policies and contextual factors on alcohol consumption and its related harms in France from 1960 to 2000. Subst Use Misuse 49:1633-45.

Com-Ruelle L, Le Guen N. (2013) Les jeunes et l'alcool: évolution des comportements, facteurs de risque et éléments protecteurs. In Quest Déconomie Santé.

Dawson DA, Goldstein RB, Patricia Chou S, et al. (2008) Age at first drink and the first incidence of adult-onset DSM-IV alcohol use disorders. Alcohol Clin Exp Res 32:2149-60.

Forouzanfar MH, Afshin A, Alexander LT et al. (2016) Global, regional, and national comparative risk assessment of 79 behavioural, environmental and occupational, and metabolic risks or clusters of risks, 1990-2015: a systematic analysis for the global burden of disease study 2015. Lancet 388: 1659-1724.

Franco S. (2015) Tackling Harmful Alcohol Use: Economics and Public Health Policy. OECD Publishing.

Gual A, Colom J. (1997) Why has alcohol consumption declined in countries of southern Europe? Addiction 92:S21-31 Wiley Online Library.

Guerin S, Laplanche A, Dunant A, et al. (2013) Alcohol-attributable mortality in France. Eur J Public Health 23:588-93.

Hingson RW, Heeren T, Winter MR. (2006) Age at drinking onset and alcohol dependence. Arch Pediatr Adolesc Med 160:8.

Human Mortality Database. (2017) University of California, Berkeley (USA), and Max Planck Institute for Demographic Research (Germany). Available at www.mortality.org or www.humanmortality.de.

Hurvich CM, Tsai C-L. (1989) Regression and time series model selection in small samples. Biometrika 76:297-307.

Hyndman RJ, Khandakar Y. (2007) Automatic time series for forecasting: the forecast package for R. Department of Econometrics and Business Statistics, Monash University.

Janssen F, Gewily SE, Bardoutsos A. (2020) Smoking epidemic in Europe in the 21st century. Tob Control (Online First: 07 August 2020) . doi: 10.1136/tobaccocontrol-2020-055658.

Janssen F, Kunst A. (2007) The choice among past trends as a basis for the prediction of future trends in old-age mortality. Popul Stud 61:315-26.

Janssen F, Kunst AE. (2004) ICD coding changes and discontinuities in trends in cause- specific mortality in six European countries, 1950-99. Bull World Health Organ 12:904-13. 
Jasilionis D, Meslé F, Shkolnikov VM, et al. (2011) Recent life expectancy divergence in Baltic countries: récentes divergences d'espérance de vie dans les pays Baltes. Eur J Popul Rev Eur Démographie 27:403-31.

Kraus L, Østhus S, Amundsen EJ, et al. (2015) Changes in mortality due to major alcohol-related diseases in four Nordic countries, France and Germany between 1980 and 2009: a comparative age-periodcohort analysis: changes in alcohol-related mortality. Addiction 110: 1443-52.

Noel JK. (2019) Associations between alcohol policies and adolescent alcohol use: a pooled analysis of GSHS and ESPAD data. Alcohol Alcohol 54:639-46.

Pitkänen T, Lyyra A-L, Pulkkinen L. (2005) Age of onset of drinking and the use of alcohol in adulthood: a follow-up study from age 8-42 for females and males. Addiction 100:652-61.

Pruckner N, Hinterbuchinger B, Fellinger M, et al. (2019) Alcohol-related mortality in the WHO European region: sex-specific trends and predictions. Alcohol Alcohol 54:593-8.

R Core Team. (2018). R: A language and environment for statistical computing. R Foundation for Statistical Computing, Vienna, Austria. https://www.Rproject.org/.

Ramstedt M. (2001) Per capita alcohol consumption and liver cirrhosis mortality in 14 European countries. Addiction 96:19-33 Wiley Online Library.

Rehm J, Gmel GE, Gmel G, et al. (2017) The relationship between different dimensions of alcohol use and the burden of disease-an update: alcohol and disease. Addiction 112:968-1001.

Rey G. (2016) Les données des certificats de décès en France: processus de production et principaux types d'analyse. Rev Médecine Interne 37: $685-93$

Rey G, Boniol M, Jougla E. (2010) Estimating the number of alcoholattributable deaths: methodological issues and illustration with French data for 2006. Addiction 105:1018-29.

Richard J-B, Palle C, Guignard R, et al. (2015) La consommation d'alcool en France en 2014. Evolutions 32:6.
Rosén M, Haglund B. (2019) Follow-up of an age-period-cohort analysis on alcohol-related mortality trends in Sweden 1970-2015 with predictions to 2025. Scand J Public Health 47:446-51.

Sheron N, Gilmore I, Parsons C, et al. (2012) Projections of alcohol-related deaths in England and Wales-tragic toll or potential prize? Lancet 379:687-8.

Sheron N, Hawkey C, Gilmore I. (2011) Projections of alcohol deaths—a wakeup call. Lancet 377:1297-9.

Stoeldraijer L, Bonneux L, van Duin C, et al. (2015) The future of smokingattributable mortality: the case of England \& Wales, Denmark and the Netherlands: the future of smoking-attributable mortality. Addiction 110:336-45.

Trias-Llimós S, Bijlsma MJ, Janssen F. (2017) The role of birth cohorts in longterm trends in liver cirrhosis mortality across eight European countries. Addiction 112:250-8.

Trias-Llimós S, Kunst AE, Jasilionis D, et al. (2018) The contribution of alcohol to the east-west life expectancy gap in Europe from 1990 onward. Int J Epidemiol 47:731-9.

Trias-Llimós S, Martikainen P, Mäkelä P, et al. (2018) Comparison of different approaches for estimating age-specific alcohol-attributable mortality: the cases of France and Finland. PLoS One 13:e0194478.

Villegas A, Kaishev VK, Millossovich P. (2015) StMoMo: an R package for stochastic mortality modelling. In 7 th Australasian Actuarial Education and Research Symposium.

WHO. (2018) Adolescent alcohol-related behaviours: trends and inequalities in the WHO European Region, 2002-2014.

WHO. (2019) Global status report on alcohol and health 2018.

WHO Global Health Observatory Data Repository. n.d. http://apps.who.i nt/gho/data/node.main.A1022?lang=en (9 November 2018, date last accessed).

Yang Y, Fu WJ, Land KC. (2004) A methodological comparison of age-periodcohort models: the intrinsic estimator and conventional generalized linear models. Sociol Methodol 34:75-110 Wiley Online Library. 